\title{
THERMALLY INDUCED VIBRATION OF AN ANNULAR PLATE SUBJECTED TO THE OSCILLATING HEAT STREAM
}

\author{
Jadwiga Kidawa-Kukla \\ Institute of Mechanics and Machine Design, Czestochowa University of Technology, Poland \\ jk@imipkm.pcz.czest.pl
}

\begin{abstract}
A solution of the problem of the thermally induced transverse vibration of an annular plate is presented. One assumes that the plate temperature changes by periodic oscillation of the heat stream in a ring region on one side of the plate. The axisymmetric temperature distribution in the plate is determined by the conductivity equation and corresponding initial and boundary conditions. The heat conduction problem in the plate and the problem of transverse vibration of the plate is solved by using the Green's function method.
\end{abstract}

\section{Introduction}

The problem of thermally induced vibration of a plate couples the heat conduction problem in the plate and the problem of mechanical vibration of this plate caused by thermal stresses. Such vibration problems are the subject of many papers (for example references [1-3]).

A study of thermally excited vibration of rectangular and circular plates was presented by Ignaczak and Nowacki in paper [1]. The considerations were confined to vibrations of plates forced by a temperature field harmonically varying with time. The solutions of the problems were found in the form of series by applying the finite sine transformation (for a rectangular plate) and the finite Hankel transformation (for circular plate). In paper [2] by Nakajo and Hayashi, the thermally induced vibrations of simply supported and clamped circular plates were considered. In the analysis, it is assumed that the distribution of temperature is linear through the thickness and along the radius. To solve this problem, authors used an analytical method and the finite element method. Within the paper [3] Kidawa-Kukla presents a solution of the problem of the thermally induced vibration of a circular plate which is subjected to the activity of a point heat source moving on the plate surface along a concentric circular trajectory.

In this paper, a solution to the problem of the thermally induced vibration of a thin annular plate is presented. The thermal moment caused by the temperature distribution in the plate is determined and displacements of the plate induced by the thermal moment are derived. The solution of the problem is obtained by using time-dependent Green's functions. 


\section{Heat conduction problem}

An annular isotropic plate of an uniform thickness $h$ with inner radius $a$ and outer radius $b$ (Fig. 1) is considered. One side of the plate is subjected by a heat souce in an annular region of the plate. The axisymmetric temperature distribution in the plate is governed by the heat conduction equation which in cylindrical coordinates is as follows

$$
\nabla^{2} T+\frac{\partial^{2} T}{\partial z^{2}}+\frac{1}{k} g(r, z, t)=\frac{1}{\kappa} \frac{\partial T}{\partial t}
$$

where $T(r, z, t)$ is temperature of the plate at the point $(r, z)$ at time $t, k$ is thermal conductivity, $\kappa$ is thermal diffusivity, $g(r, z, t)$ represents a heat generation term and $\nabla^{2}=\frac{\partial^{2}}{\partial r^{2}}+\frac{1}{r} \frac{\partial}{\partial r}$. The heat generation term is assumed in the form

$$
g(r, z, t)=\varphi(t) \theta(r) \delta(z-h)
$$

where $\theta$ characterises the stream of the heat, $\delta()$ is the Dirac delta function, $\varphi(t)$ is the function describing the change with time of the heat stream

$$
\varphi(t)=\sin \omega t
$$

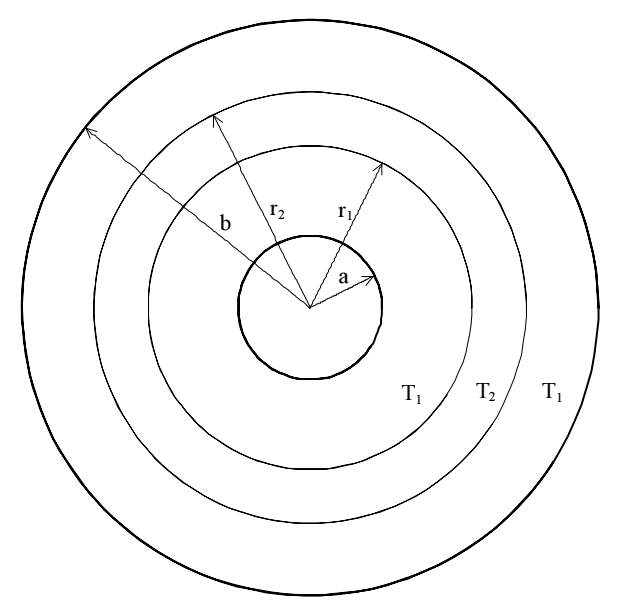

Fig. 1. A schema of an annular plate

Moreover, the following initial and boundary conditions are assumed:

$$
T(r, z, 0)=0
$$




$$
\begin{gathered}
\left.\frac{\partial T(r, z, t)}{\partial r}\right|_{r=a}=0,\left.\frac{\partial T(r, z, t)}{\partial r}\right|_{r=b}=0 \\
k \frac{\partial T}{\partial z}(r, h, t)=\alpha_{0}\left[T_{0}-T(r, h, t)\right] \\
k \frac{\partial T}{\partial z}(r, 0, t)=\alpha_{0}\left[T_{0}-T(r, 0, t)\right]
\end{gathered}
$$

where $\alpha_{0}$ is a heat transfer coefficient, $T_{0}$ is the known temperature of a surrounding medium.

The solution of the problem (1) and (4)-(7) can be presented in the form [1]

$$
T(r, z, t)=\frac{\kappa}{k} \int_{\tau=0}^{t} \int_{z^{\prime}=0}^{h} \int_{r^{\prime}=a}^{b} r G\left(r, z, t ; r^{\prime}, z^{\prime}, \tau\right) g\left(r^{\prime}, z^{\prime}, \tau\right) d r^{\prime} d z^{\prime} d \tau
$$

where $G$ is the Green's function (GF) corresponding to the problem of the heat conduction in cylindrical coordinates. Taking into account the function $g$ given by (2) in equation (8), one obtains

$$
T(r, z, t)=\frac{\kappa}{k} \int_{\tau=0}^{t} \int_{r^{\prime}=a}^{b} r^{\prime} G\left(r, z, t ; r^{\prime}, h, \tau\right) \varphi(\tau) \theta\left(r^{\prime}\right) d r^{\prime} d \tau
$$

The Green's function $G$ satisfies the zero initial and boundary conditions analogous to the conditions (4)-(7). The GF for annulus with radial and angular dependence is given in paper [3]. For the considered axisymmetric case of annular plate the needed GF can be constructed as a product of two one-dimensional GFs, i.e.

$$
G\left(r, z, t ; r^{\prime}, z^{\prime}, \tau\right)=G_{R}\left(r, t ; r^{\prime}, \tau\right) G_{Z}\left(z, t ; z^{\prime}, \tau\right)
$$

The Green's function $G_{R}$ can be written in the form [4]

$$
G_{R}\left(r, t ; r^{\prime}, \tau\right)=\frac{1}{\pi\left(b^{2}-a^{2}\right)}-\frac{\pi}{4 a^{2}} \sum_{m=1}^{\infty} \exp \left(-\alpha \beta_{m}^{2} \frac{t-\tau}{a^{2}}\right) \frac{\beta_{m}^{2} J_{1}^{2}\left(\beta_{m}\right) \phi_{m}(r) \phi_{m}\left(r^{\prime}\right)}{J_{1}^{2}\left(\beta_{m}\right)-J_{1}^{2}\left(\beta_{m} \frac{b}{a}\right)}
$$

where

$$
\phi_{m}(u)=J_{0}\left(\beta_{m} \frac{u}{a}\right) Y_{1}\left(\beta_{m} \frac{b}{a}\right)-J_{1}\left(\beta_{m} \frac{b}{a}\right) Y_{0}\left(\beta_{m} \frac{u}{a}\right)
$$

and $\beta_{m}(m=1,2, \ldots)$ are roots of the equation 


$$
J_{1}\left(\beta_{m}\right) Y_{1}\left(\beta_{m} \frac{b}{a}\right)-J_{1}\left(\beta_{m} \frac{b}{a}\right) Y_{0}\left(\beta_{m}\right)=0
$$

The Green's function $G_{Z}$ has the form [4]

$$
G_{Z}\left(z, t ; z^{\prime}, \tau\right)=\frac{2}{h} \sum_{n=1}^{\infty} \exp \left(-\alpha \gamma_{n}^{2} \frac{t-\tau}{h^{2}}\right) \psi_{n}(z) \psi_{n}\left(z^{\prime}\right)
$$

where $B_{1}=\frac{\alpha_{0} h}{k}, B_{2}=\frac{\alpha_{1} h}{k}$,

$$
\psi_{n}(u)=\left[\gamma_{n} \cos \left(\gamma_{n} \frac{u}{h}\right)+B_{1} \sin \left(\gamma_{n} \frac{u}{h}\right)\right]\left\{\left(\gamma_{n}^{2}+B_{1}^{2}\right)\left[1+B_{2} /\left(\gamma_{n}^{2}+B_{2}^{2}\right)\right]+B_{1}\right\}^{-\frac{1}{2}}
$$

and $\gamma_{n}(\mathrm{n}=1,2, \ldots)$ are roots of the equation

$$
\left(\gamma_{n}^{2}-B_{1} B_{2}\right) \sin \left(\gamma_{n}\right)-\gamma_{n}\left(B_{1}+B_{2}\right) \cos \left(\gamma_{n}\right)=0
$$

The temperature distribution in the plate is obtained by substituting (10)-(11) and (14) in equation (9)

$$
\begin{aligned}
& T(r, z, t)=\frac{\kappa}{k}\left\{\frac{2}{\pi h^{2}\left(b^{2}-a^{2}\right)} \int_{r^{\prime}=a}^{b} r^{\prime} \theta\left(r^{\prime}\right) d r^{\prime} \cdot \sum_{n=1}^{\infty} P_{0 n}(t) \psi_{n}(z) \psi_{n}(h)\right. \\
& -\frac{\pi}{2 \alpha a^{2} h} \sum_{m=1}^{\infty} \sum_{n=1}^{\infty} P_{m n}(t) \int_{r^{\prime}=a}^{b} r^{\prime} \theta\left(r^{\prime}\right) \varphi_{m}\left(r^{\prime}\right) d r^{\prime} \cdot \frac{\beta_{m}^{2} J_{1}^{2}\left(\beta_{m}\right) \varphi_{m}(r) \psi_{n}(z) \psi_{n}(h)}{J_{1}^{2}\left(\beta_{m}\right)-J_{1}^{2}\left(\beta_{m} \frac{b}{a}\right)}
\end{aligned}
$$

where (it is assumed: $\beta_{0}=0$ )

$$
P_{m n}(t)=\int_{\tau=0}^{t} \varphi(\tau) \exp \left(-\alpha\left(\frac{\beta_{m}^{2}}{a^{2}}+\frac{\gamma_{n}^{2}}{h^{2}}\right)(t-\tau)\right) d \tau \quad \text { for } m=0,1,2, \ldots, n=1,2, \ldots
$$

For $\varphi(\tau)=\sin \omega \tau$, we have

$$
P_{m n}(t)=\frac{1}{d^{2}+m^{2}}\left(e^{-b t} m-m \cos m t+d \sin m t\right)
$$

where $d=\alpha\left(\frac{\beta_{m}^{2}}{a^{2}}+\frac{\gamma_{n}^{2}}{h^{2}}\right)$. 


\section{The problem of vibration of the plate thermally induced by sinusoidally varying heat stream}

The vibration of the considered plate is governed by the differential equation

$$
D \nabla^{4} w+\mu \frac{\partial^{2} w}{\partial t^{2}}=-\nabla^{2} M_{T}
$$

where $D$ is a flexural stiffness, $\mu$ is a mass per unit area of the plate, $w(r, \Phi, t)$ is a displacement of the middle surface of the plate at the point $(r, \Phi)$ at time $t$, and $M_{T}$ is a thermal moment. The thermal moment is defined as [1]

$$
M_{T}=\frac{\alpha E}{1-v} \int_{0}^{h} z T(r, \phi, z, t) d z
$$

The presented study deals with the annular plate with simply supported edges, which means that the following boundary conditions are satisfied

$$
w=0, \quad-D\left[\frac{\partial^{2} w}{\partial r^{2}}+v\left(\frac{1}{r} \frac{\partial w}{\partial r}+\frac{1}{r^{2}} \frac{\partial^{2} w}{\partial \phi^{2}}\right)\right]=0 \quad \text { at } r=a \text { and } r=b
$$

Moreover, the zero initial conditions are assumed

$$
w=\frac{\partial w}{\partial t}=0 \quad \text { for } t=0
$$

Taken into account equation (17) and (20), we obtain the thermal moment

$$
\begin{aligned}
& M_{T}(r, t)=\frac{\alpha \kappa E}{(1-v) k}\left\{\frac{2}{\pi h^{2}\left(b^{2}-a^{2}\right) \int_{r^{\prime}=a}^{b} r^{\prime} \theta\left(r^{\prime}\right) d r^{\prime}}\right. \\
& \cdot \sum_{n=1}^{\infty} P_{0 n}(t) \frac{A_{n} \psi_{n}(h)}{\left\{\left(\gamma_{n}^{2}+B_{1}^{2}\right)\left[1+B_{2} /\left(\gamma_{n}^{2}+B_{2}^{2}\right)\right]+B_{1}\right\}^{\frac{1}{2}}}+ \\
& -\frac{\pi}{2 \alpha a^{2} h} \sum_{m=1}^{\infty} \sum_{n=1}^{\infty} P_{m n}(t) \int_{r^{\prime}=a}^{b} r^{\prime} \theta\left(r^{\prime}\right) \phi_{m}\left(r^{\prime}\right) d r^{\prime} \\
& \left.\cdot \frac{\beta_{m}^{2} J_{1}^{2}\left(\beta_{m}\right) \phi_{m}(r)}{J_{1}^{2}\left(\beta_{m}\right)-J_{1}^{2}\left(\beta_{m} \frac{b}{a}\right)} \cdot \frac{A_{n} \psi_{n}(h)}{\left\{\left(\gamma_{n}^{2}+B_{1}^{2}\right)\left[1+B_{2} /\left(\gamma_{n}^{2}+B_{2}^{2}\right)\right]+B_{1}\right)^{\frac{1}{2}}}\right\}
\end{aligned}
$$


where

$$
A_{n}=h^{2}\left[\sin \left(\gamma_{n}\right)-\frac{B_{1}}{\gamma_{n}} \cos \left(\gamma_{n}\right)\right]+\frac{h^{2}}{\gamma_{n}}\left[\cos \left(\gamma_{n}\right)+\frac{B_{1}}{\gamma_{n}} \sin \left(\gamma_{n}\right)-1\right]
$$

The solution to the vibration problem (19), (21)-(22) of the annular plate can be expressed in the form

$$
w(r, \phi, t)=\int_{0}^{t} \int_{0}^{b} \nabla^{2} M_{T}(\rho, \tau) G_{v}(r, t ; \rho, \tau) d \rho d \tau
$$

where $G_{v}$ is the Green's function corresponding to the considered vibration problem.

\section{The Green's function}

The differential equation for the Green's function $G(r, t ; \rho, \tau)$ has the form

$$
\left(\frac{\partial^{2}}{\partial r^{2}}+\frac{1}{r} \frac{\partial}{\partial r}\right)^{2} G_{v}+\frac{\mu}{D} \frac{\partial^{2} G_{v}}{\partial t^{2}}=\frac{\delta(r-\rho) \delta(t-\tau)}{2 \pi D r}
$$

The function $G_{v}$ satisfies the zero initial and homogeneous boundary conditions analogous to the conditions (21)-(22), i.e.

$$
\begin{gathered}
G_{v}(a, t)=0,\left.\left[\frac{\partial^{2} G_{v}}{\partial r^{2}}+\frac{v}{r} \frac{\partial G_{v}}{\partial r}\right]\right|_{r=a}=0 \\
G_{v}(b, t)=0,\left.\quad\left[\frac{\partial^{2} G_{v}}{\partial r^{2}}+\frac{v}{r} \frac{\partial G_{v}}{\partial r}\right]\right|_{r=b}=0 \\
G_{v}(r, 0)=0,\left.\quad \frac{\partial G_{v}}{\partial t}\right|_{t=0}=0
\end{gathered}
$$

The solution of the initial-boundary problem (26)-(28) can be presented in the form

$$
G_{v}(r, t)=\sum_{n=1}^{\infty} Q_{n}(r) \Gamma_{n}(t)
$$

where $Q_{m n}(r)$ are the eigenfunctions of the following boundary problem 


$$
\begin{gathered}
{\left[\frac{d^{2}}{d r^{2}}+\frac{1}{r} \frac{d}{d r}\right]^{2} Q_{n}(r)-\lambda_{n}^{4} Q_{n}(r)=0} \\
Q_{n}(a)=0,\left.\left[\frac{\partial^{2} Q_{n}}{\partial r^{2}}+\frac{v}{r} \frac{\partial Q_{n}}{\partial r}\right]\right|_{r=a}=0 \\
Q_{n}(b)=0,\left.\left[\frac{\partial^{2} Q_{n}}{\partial r^{2}}+\frac{v}{r} \frac{\partial Q_{n}}{\partial r}\right]\right|_{r=b}=0
\end{gathered}
$$

The general solution of the differential equation (30) can be written in the form

$$
Q_{n}(r)=C_{1} J_{0}\left(\lambda_{n} r\right)+C_{2} Y_{0}\left(\lambda_{n} r\right)+C_{3} I_{0}\left(\lambda_{n} r\right)+C_{4} K_{0}\left(\lambda_{n} r\right)
$$

where $J_{0}, Y_{0}$ are the Bessel functions of order zero, and $I_{0}, K_{0}$ are the modified Bessel functions of order zero. Substituting the function (31) into the boundary conditions (31)-(32), we obtain a system of homogeneous equations

$$
\sum_{j=1}^{4} a_{i j} C_{j}=0, \quad i=1, \ldots, 4
$$

where:

$$
\begin{array}{cll}
A_{11}=J_{0}\left(\lambda_{n} a\right), \quad A_{12}=Y_{0}\left(\lambda_{n} a\right), & A_{13}=I_{0}\left(\lambda_{n} a\right), \quad A_{14}=K_{0}\left(\lambda_{n} a\right) \\
A_{21}=J_{0}\left(\lambda_{n} b\right), \quad A_{22}=Y_{0}\left(\lambda_{n} b\right), & A_{23}=I_{0}\left(\lambda_{n} b\right), \quad A_{24}=K_{0}\left(\lambda_{n} b\right) \\
A_{31}=-\lambda_{n}^{2} J_{0}\left(\lambda_{n} a\right)+\frac{1-v}{a} \lambda_{n} J_{1}\left(\lambda_{n} a\right), & A_{32}=-\lambda_{n}^{2} Y_{0}\left(\lambda_{n} a\right)+\frac{1-v}{a} \lambda_{n} Y_{1}\left(\lambda_{n} a\right) \\
A_{33}=\lambda_{n}^{2} I_{0}\left(\lambda_{n} a\right)-\frac{1-v}{a} \lambda_{n} I_{1}\left(\lambda_{n} a\right), & A_{34}=\lambda_{n}^{2} K_{0}\left(\lambda_{n} a\right)+\frac{1-v}{a} \lambda_{n} K_{1}\left(\lambda_{n} a\right) \\
A_{41}=-\lambda_{n}^{2} J_{0}\left(\lambda_{n} b\right)+\frac{1-v}{b} \lambda_{n} J_{1}\left(\lambda_{n} b\right), & A_{42}=-\lambda_{n}^{2} Y_{0}\left(\lambda_{n} b\right)+\frac{1-v}{b} \lambda_{n} Y_{1}\left(\lambda_{n} b\right) \\
A_{43}=\lambda_{n}^{2} I_{0}\left(\lambda_{n} b\right)-\frac{1-v}{b} \lambda_{n} I_{1}\left(\lambda_{n} b\right), & A_{44}=\lambda_{n}^{2} K_{0}\left(\lambda_{n} b\right)+\frac{1-v}{b} \lambda_{n} K_{1}\left(\lambda_{n} b\right)
\end{array}
$$

The non-trivial solution of the system (32) exists for these $\lambda_{n}$, which satisfy the equation

$$
\operatorname{det}\left[A_{i j}\right]=0
$$


Roots of the equation (35) are determined numerically. The eigenfunctions corresponding to the roots are derived by substituting a solution of the system (34) in the equation (33). After transformations, the eigenfunctions can be presented in the form

$$
Q_{n}(r)=\bar{C}_{1} J_{0}\left(\lambda_{n} r\right)+\bar{C}_{2} Y_{0}\left(\lambda_{n} r\right)+\bar{C}_{3} I_{0}\left(\lambda_{n} r\right)+\bar{C}_{4} K_{0}\left(\lambda_{n} r\right)
$$

where

$$
\begin{gathered}
\bar{C}_{1}=2 \lambda_{n}^{2} \psi_{I K} Y_{0}\left(a \lambda_{n}\right)+\frac{1-v}{a} \lambda_{n}\left[\psi_{I Y} K_{1}\left(a \lambda_{n}\right)-\psi_{I K} Y_{1}\left(a \lambda_{n}\right)-\psi_{K Y} I_{1}\left(a \lambda_{n}\right)\right] \\
\bar{C}_{2}=-2 \lambda_{n}^{2} \psi_{I K} J_{0}\left(a \lambda_{n}\right)+\frac{1-v}{a} \lambda_{n}\left[\psi_{I K} J_{1}\left(a \lambda_{n}\right)-\psi_{J K} I_{1}\left(a \lambda_{n}\right)+\psi_{J I} K_{1}\left(a \lambda_{n}\right)\right] \\
\bar{C}_{3}=-2 \lambda_{n}^{2} \psi_{J Y} K_{0}\left(a \lambda_{n}\right) \\
-\frac{1-v}{a} \lambda_{n}\left[2 K_{0}\left(b \lambda_{n}\right)+a \lambda_{n} \pi\left(\psi_{J Y} K_{1}\left(a \lambda_{n}\right)+\left(J_{0}\left(b \lambda_{n}\right) Y_{1}\left(a \lambda_{n}\right)-J_{1}\left(a \lambda_{n}\right) Y_{0}\left(b \lambda_{n}\right)\right) K_{0} a \lambda_{n}\right)\right] \\
\bar{C}_{4}=-2 \lambda_{n}^{2} \psi_{J Y} I_{0}\left(a \lambda_{n}\right)-\frac{1-v}{a} \lambda_{n}\left[\psi_{I Y} J_{1}\left(a \lambda_{n}\right)-\psi_{J Y} I_{1}\left(a \lambda_{n}\right)+\psi_{J I} Y_{1}\left(a \lambda_{n}\right)\right]
\end{gathered}
$$

and

$$
\begin{aligned}
& \psi_{J Y}=J_{0}\left(a \lambda_{n}\right) Y_{0}\left(b \lambda_{n}\right)-J_{0}\left(b \lambda_{n}\right) Y_{0}\left(a \lambda_{n}\right) \\
& \psi_{I Y}=I_{0}\left(a \lambda_{n}\right) Y_{0}\left(b \lambda_{n}\right)-I_{0}\left(b \lambda_{n}\right) Y_{0}\left(a \lambda_{n}\right) \\
& \psi_{J I}=J_{0}\left(a \lambda_{n}\right) I_{0}\left(b \lambda_{n}\right)-J_{0}\left(b \lambda_{n}\right) I_{0}\left(a \lambda_{n}\right) \\
& \psi_{I K}=I_{0}\left(a \lambda_{n}\right) K_{0}\left(b \lambda_{n}\right)-I_{0}\left(b \lambda_{n}\right) K_{0}\left(a \lambda_{n}\right) \\
& \psi_{K Y}=K_{0}\left(a \lambda_{n}\right) Y_{0}\left(b \lambda_{n}\right)-K_{0}\left(b \lambda_{n}\right) Y_{0}\left(a \lambda_{n}\right) \\
& \psi_{J K}=J_{0}\left(a \lambda_{n}\right) K_{0}\left(b \lambda_{n}\right)-J_{0}\left(b \lambda_{n}\right) K_{0}\left(a \lambda_{n}\right)
\end{aligned}
$$

Note that the functions $R_{m n}$ satisfy the orthogonality condition

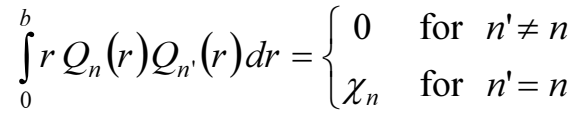

where

$$
\begin{aligned}
\chi_{m}(\lambda)=\frac{b^{2}}{2 \lambda}\left[J_{m}^{2}(\lambda) I_{m-1}(\lambda)\left(-\lambda I_{m-1}(\lambda)+2(m-1) J_{m}(\lambda)\right)\right. \\
\left.I_{m}^{2}(\lambda) J_{m-1}(\lambda)\left(\lambda J_{m-1}(\lambda)-2(m-1) J_{m}(\lambda)\right)+2 \lambda J_{m}^{2}(\lambda) I_{m}^{2}(\lambda)\right]
\end{aligned}
$$


Taking into account (23)-(24) and using the orthogonality condition (37) in equations (20) and (22), we obtain the differential equation

$$
\frac{\partial^{2} \Gamma_{n}(t)}{\partial t^{2}}+\frac{D}{b^{4} \mu} \lambda_{n}^{4} \Gamma_{n}(t)=\frac{Q_{n}(\rho)}{2 \pi \mu \chi_{m}\left(\lambda_{m n}\right)} \delta(t-\tau)
$$

and initial conditions

$$
\Gamma_{n}(0)=0,\left.\quad \frac{d \Gamma_{n}}{d t}\right|_{t=0}=0
$$

The solution of the initial problem (39)-(40) has the form

$$
\Gamma_{n}(t)=\frac{Q_{n}(\rho)}{2 \pi \mu \Omega_{n} \chi_{n}} \sin \Omega_{n}(t-\tau) H(t-\tau)
$$

where $\Omega_{n}^{2}=\frac{D}{b^{4} \mu} \lambda_{n}^{4}$ and $H$ denotes the Heaviside function.

Finally, on the basis of equations (29), (36), (41), the Green's function for the simply supported annular plate can be written in the following form

$$
G_{v}(r, t ; \rho, \tau)=\frac{H(t-\tau)}{2 \pi \mu} \sum_{n=1}^{\infty} \frac{Q_{n}(\rho)}{\Omega_{n}} Q_{n}(r) \sin \Omega_{n}(t-\tau)
$$

Taking into account equations (23) and (42) in equation (24), we obtain the displacements of the plate caused by the thermal moment.

\section{Conclusions}

The solution of the problem of transverse vibration of an annular plate, thermally induced by a heat stream which acts on a concentric annular part of the plate and changes with time sinusoidally, was presented. The problem was based on the differential equations of the heat conduction and the transverse vibration of the plate, which were complemented by initial and boundary conditions. The temperature distribution and the transverse deflection of the annular plate in an analytical form were obtained by using the properties of the Green's function. The presented solution can be used for numerical investigation of the thermally induced vibration of the plate.

\section{References}

[1] Ignaczak J., Nowacki W., Transversal vibration of a plate, produced by heating, Archives de Mecanique Appliquee 1961, XIII (5), 650-667. 
[2] Nakajo Y., Hayashi K., Response of simply supported and clamped circular plates to thermal impact, Journal of Sound and Vibration 1988, 122(2), 347-356.

[3] Kidawa-Kukla J., Application of the Green's function method to the problem of thermally induced vibration of a circular plate, Scientific Research of the Institute of Mathematics and Computer Science 2010, 1(9), 53-60.

[4] Beck J.V. et al., Heat Conduction Using Green's Functions, Hemisphere Publishing Corporation, London 1992.

[5] Duffy D.G., Green's Functions with Applications - Studies in Advanced Mathematics, Boca Raton, London, New York 2001. 